\title{
Low-Energy Secondary Electron Filtering with Immersion Lens SEM
}

\author{
Richard Young,* Eric Bosch,** Marek Uncovsky,*** and Lubos Tuma*** \\ *FEI Company, 5350 NE Dawson Creek Drive, Hillsboro, OR 97124, USA \\ **FEI Company, Building AAE, PO Box 80066, 5600 KA Eindhoven, The Netherlands \\ ***FEI Company, Technologicky Park, Podnikatelska 6, Brno, Czech Republic
}

The scanning electron microscope (SEM) offers a rich array of different electron-beam induced signals with which to create the final micrograph. So in parallel with efforts to improve the beam resolution [1], it is also important to find ways to make the best use of these induced signals to bring out the desired information about the sample. As an example of this effort, this paper will describe the use of energy filtering of the secondary electron (SE) signal using an immersion lens SEM. Applications of this technique include visualizing dopant contrast [2] and improving the sensitivity of passive voltage contrast, Figure 1, as well as the potential for increasing the contrast between key materials of interest by exploiting the different SE energy distributions from different materials (see for example, [3] for experimental measurements of SE spectra).

To better understand the complete imaging process Monte-Carlo simulations have been undertaken that include a 3D modeling of the SEM column, including the electromagnetic fields, so that the trajectories of the emitted SE and back-scattered electrons (BSE) from the beam-sample interaction can be accurately followed back into the SEM detection system [4]. Once such a model is in place virtual experiments can be carried to determine the optimum beam and detector conditions for a particular sample type. Such simulations can investigate the signal based on particular SE energies or trajectory angles from the sample, and also to simulate the actual image expected.

This modeling has been applied to the energy filtering of the SE signal. Low-pass, high-pass and band-pass filtering can be carried out depending upon the beam, sample and detector conditions. In each case, the magnetic field of the immersion lens SEM combined with an in-lens detector play the crucial role in enabling the filtering. Figure 2 shows modeled results for a low pass filter where with in-lens detector parameter $\mathrm{M}=-2 \mathrm{~V}$ the electrons up to $\sim 5 \mathrm{eV}$ are transmitted, while a value of $\mathrm{M}=-16 \mathrm{~V}$ allows a wider range $(\sim 16 \mathrm{eV})$. The modeling showed that by adjustment of $\mathrm{M}$ it was possible to go from zero to full transmission of the SE range, with Fig.2 just being representative examples. Figure 1 shows experimental results for a high pass filter on a voltage contrast sample by adjusting the starting point of the range from $1 \mathrm{eV}$ to $2 \mathrm{eV}$ it was possible to change which of the contacts appear bright or dark in the image.

References

[1] R.J. Young et al, Microscopy Today, Vol 16, Number 4 (2008) 24

[2] P. Kazemian et al, Ultramicroscopy 107 (2007) 140-150

[3] D.C. Joy et al, J. Microsc. 215 (2004) 77

[4] E. Kieft and E. Bosch, J. Phys. D: Appl. Phys. 41 (2008) 215310 

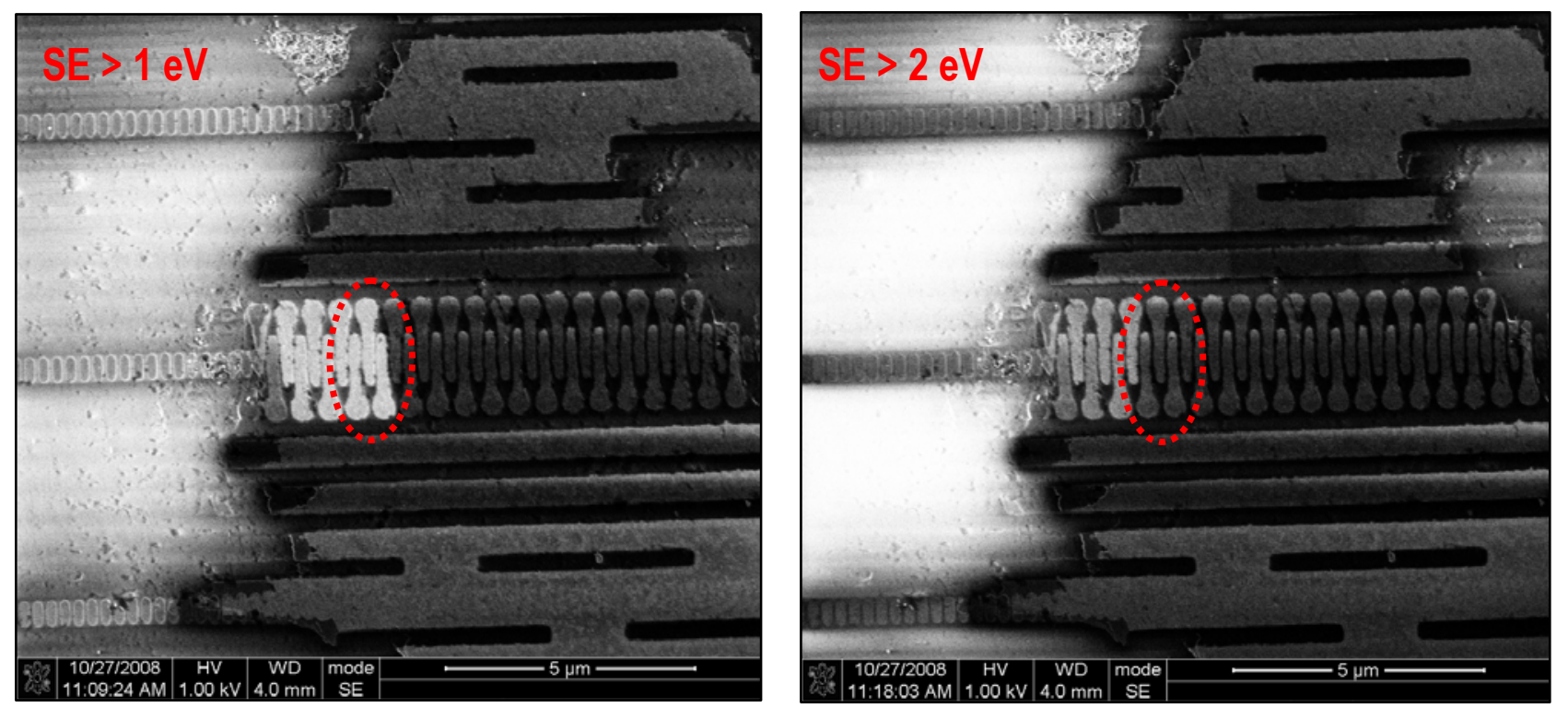

Fig. 1. High-pass filtered images of exposed contacts on a deprocessed memory array. Note contrast change in several of the contacts (see circled areas) between the left image (high pass filter $>1 \mathrm{eV}$ ) and the right image $(>2 \mathrm{eV})$. The $1 \mathrm{eV}$ and $2 \mathrm{eV}$ values are based on the modeled filter performance.
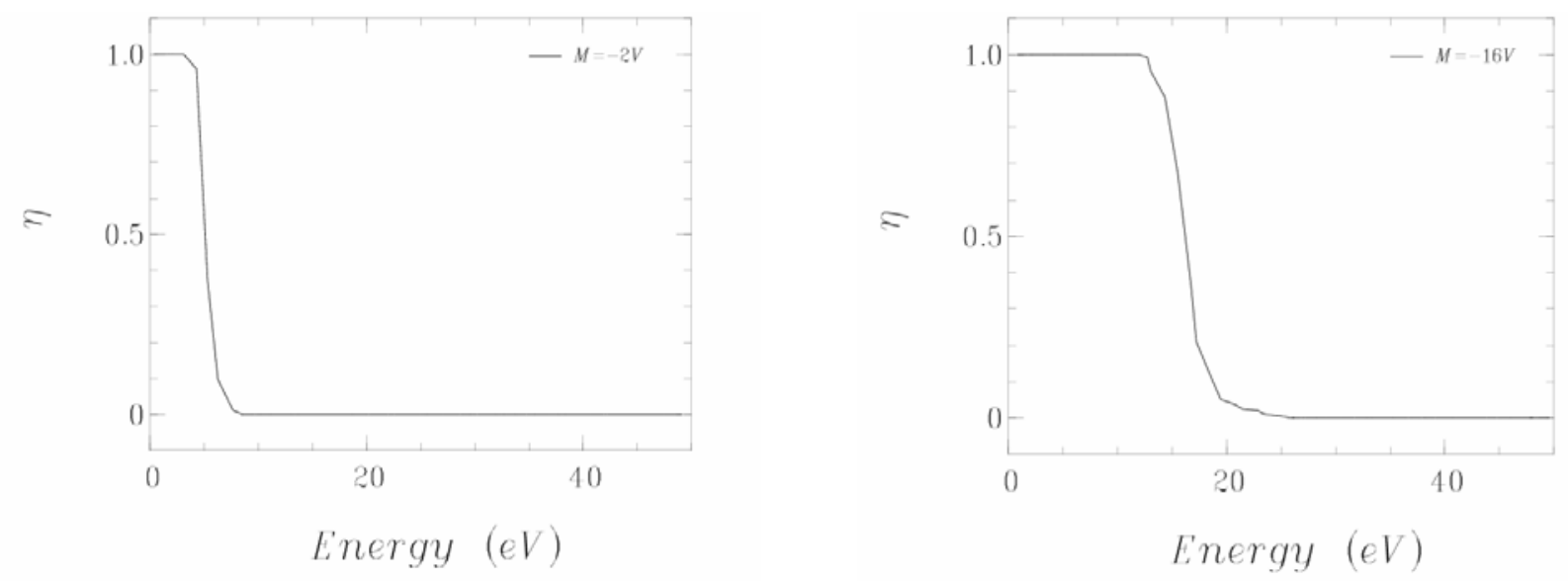

Fig. 2. Low pass filtering of SE signal from Monte-Carlo modeling. The graphs show collected signal versus SE energy for two different detector settings (beam energy $1 \mathrm{keV}$ ). With parameter $\mathrm{M}=-2 \mathrm{~V}$ the electrons up to $\sim 5 \mathrm{eV}$ are transmitted, while a value of $\mathrm{M}=-16 \mathrm{~V}$ allows $\sim 16 \mathrm{eV}$ range. 